\title{
Study on the Clinicopathological Features and Prognostic Factors of Extranodal NK/T Cell Lymphoma, Nasal Type (with 18 cases) \\ S Yang ${ }^{123}$, T Jiang ${ }^{1}, \mathrm{~J}$ Dong $^{4}$, Qian Xu ${ }^{1}$, Zhangchi Pan ${ }^{1}, \mathrm{~J} \mathrm{Wang}^{1}$, Q Chen ${ }^{123}$
}

\begin{abstract}
Aim. The study is to analyze the clinicopathological features of extranodal NK/T cell lymphoma, nasal type (ENKCL) and its treatment and efficacy, to provide a scientific basis for individualized treatment.

Methods. We conducted a retrospective analysis of the clinicopathological records of 18 ENKCL cases treated in Union Hospital of Fujian Medical University from May 2007 to May 2012, with follow-up of patients and univariate and multivariate analysis of the clinicopathological parameters, treatment and 2-year survival rate.

Results. Among the 18 ENKCL cases, patients' age ranged from 20 to 69, with an average age of 41.3 and male/female ratio of 7/1. The 2-year overall survival (OS) rate in Ann Arbor Stage I/ II patients with ECOG PS $<2$, over 2 sites of extranodal involvement, no bone marrow infiltration and international prognostic index (IPI) $\leq 2$ was significantly higher than Ann Arbor Stage II/IV patients with ECOG PS $\geq 2$, less than 2 extranodal involvement sites, bone marrow infiltration and IPI $>2$. The 2-year OS rate of patients with white blood cell count (before treatment) $\geq 4.0 \times 10^{9} / \mathrm{L}$, hemoglobin value $\geq 120 \mathrm{~g} / \mathrm{L}$ and normal lactate dehydrogenase level was significantly higher than patients with white blood cell count (before treatment) $<4.0 \times 10^{9} / \mathrm{L}$, hemoglobin $<120$ $\mathrm{g} / \mathrm{L}$ and elevated lactate dehydrogenase level. Patients' age, gender, B symptoms and albumin level in blood before treatment were not related to prognosis.
\end{abstract}

Keywords: Diagnosis, extranodal NK/T cell lymphoma, nasal type, prognosis, treatment

From: ${ }^{1}$ Teaching and Research Section of Oncology, Union Clinical Medical School of Fujian Medical University, ${ }^{2}$ Department of Oncology, Union Hospital of Fujian Medical University, ${ }^{3}$ Fujian Provincial Key Laboratory of Translational Cancer Medicine, ${ }^{4}$ Teaching and Research Section of Hematology, Union Hospital of Fujian Medical University ${ }^{4}$, Fuzhou 350001, PR China.

Correspondence: Dr S Yang, Teaching and Research Section of Oncology, Union Clinical Medical School of Fujian Medical University, China. E-mail: drcqiang@126.com 


\section{Extranodal NK/T Cell Lymphoma}

After receiving the first course of chemotherapy, radiotherapy or both, 11 out of the 18 patients achieved complete remission and 7 did not and the 2-year OS rate of the former was significantly higher than that of the latter. 15 patients received chemotherapy alone or chemotherapy and radiotherapy combined. The 2-year OS rate of those who were treated with first-line regimen as classic CHOP were significantly improved compared with those receiving other treatments. Among the 8 patients treated with only chemotherapy, 4 of them received 1-2 cycles of therapy and 4 went through over 2 cycles, and the 2-year OS rate of the two showed no statistically significant difference. Immunohistochemical results suggested that the 2-year OS rates in patients with NK cell phenotype, negative or low CD56 expression and Ki-67 proliferation index higher than 30\% was significantly increased compared with those with T cell phenotype, medium or high CD56 expression and Ki-67 expression lower than 30\%. Multivariate analysis indicated that tumor stage, ECOG PS score, the number of sites of extranodal involvement, bone marrow infiltration, IPI score, white blood cell count before treatment, hemoglobin value, lactate dehydrogenase level, efficacy of first treatment, CD56 and Ki-67 expression, and lymphocyte phenotype are independent prognostic factors for ENKCL.

Conclusion. ENKCL lesions develop rapidly with poor prognosis. Ann Arbor staging, ECOG PS score, the number of extranodal involvement sites, bone marrow infiltration, IPI score, white blood cell count before treatment, hemoglobin value, lactate dehydrogenase level, the efficacy of first treatment, CD56 and Ki-67 expression, and lymphocyte phenotype are independent prognostic factors, correlated with prognosis and survival and important for clinical diagnosis and treatment. Though today we promote the comprehensive treatment of ENKCL, chemotherapy is still of great importance. Individualized treatment needs to be further studied and prognostic indicators of greater clinicopathological significance for ENKCL are worthy of further exploration. 


\section{INTRODUCTION}

Extranodal NK/T cell lymphoma, nasal type (ENKCL), recently recognized as a non-Hodgkin's lymphoma (NHL), demonstrates distinct regional and gender differences in the incidence of the disease. It is most commonly found in Asia and South America and quite rare in North America and European countries. It accounts for 2\%-10\% of all non-Hodgkin's lymphoma. It is more common in men than in women $(1,2)$. ENKCL presents unique clinical features, strong invasiveness, rapid progression, high mortality and poor prognosis. Its pathogenesis has not yet been fully understood. There remains disagreement regarding its treatment and prognosis and a lack of effective measures in its prevention and treatment $(3,4)$. Compared with B-cell lymphoma, ENKCL requires more in-depth research, particularly in more precise individualized treatment clinically. This study analyzes the clinicopathological features of ENKCL and discusses the correlation between the pathological factors and the prognosis with literature study in order to provide guidance for clinical treatment.

\section{MATERIALS AND METHODS}

\section{Subjects}

We collected the clinical and pathological data of 18 ENKCL cases confirmed by the pathomorphology and immunohistochemical enzyme treated in Union Hospital of Fujian Medical University from May 2007 to May 2012, including gender, age, Ann Arbor staging, ECOG PS score, the number of sites of extranodal involvement, B symptoms, bone marrow infiltration, international prognostic index (IPI) score, and white blood cell count before treatment, hemoglobin value, albumin concentration, lactate dehydrogenase level, 
pathological biopsy, immunohistochemical results, efficacy of first treatment, treatment options, survival rate, etc., to conduct retrospective analysis of its clinical and pathological features.

\section{Pathological diagnosis and immunohistochemical detection}

Pathological diagnosis was performed using paraffin-embedded sections, hematoxylin and eosin stain and histomorphological examination according to WHO criteria for lymphoid and hematopoietic malignancies (5). Immunohistochemical staining was conducted using Elivion TM plus method with antibodies and kits purchased from Fuzhou Maixin Company according to the instruction on the kits. PBS, instead of primary antibody, was used for negative control and positive control was designed. In all cases antibody tests were conducted, including CD2, CD3, CD20, CD43, CD56, CD45RO, CD117, CK, BCL-6, GranzymeB, EBER, LCA, TIA-1, Ki-67, etc., and results were analyzed with reference to related literature (6). Positive CD56 expression showed membrane stained brown yellow or brown and results were classified by color. They were classified into four groups:"-" (no stained cells); "+" (fraction of stained cells $<25 \%$ ); "++" (fraction of stained cells ranged from $25 \%$ to $50 \%$ ); " +++ " (fraction of stained cells $\geq 50 \%$ ). Positive Ki-67 expression showed brown yellow or brown granular precipitate within the nucleus. When observing, we selected 5 high-power fields (400x) in each slice, counted 200 cancer cells in each field and calculated the proportion of positive cells as Ki-67 labeling index $(7,8)$. ENKCL was identified as NK cell

phenotype with immunohistochemical expression of $\mathrm{CD}_{2}, \mathrm{CD}_{3 \varepsilon}, \mathrm{CD}_{56}$ and other indicators being positive and $\mathrm{T}$ cell phenotype with immunohistochemical expression of $\mathrm{CD}_{3 \varepsilon}, \mathrm{CD}_{43}$, 
$\mathrm{CD}_{45 \mathrm{RO}}$ and other indicators being positive $(9,10)$.

\section{Clinical examination and clinical stage}

Clinical examination included a detailed physical examination, endoscopy, imaging, cytological examination of bone marrow, etc. Staging criteria is strictly in accordance with Ann Arbor staging criteria of American Joint Committee on Cancer (AJCC) and Union for International Cancer Control (UICC). B symptoms involved unexplained fever (often body temperature above $38^{\circ} \mathrm{C}$ ) within six months, night sweats and weight loss (of $10 \%$ or more). IPI, including age, tumor stage, ECOG PS score, number of sites of extranodal involvement and serum lactate dehydrogenase level before treatment, was divided into low risk / low-medium risk group (IPI: 0-2) and high-risk group (IPI: 3-5). Short-term efficacy was evaluated using the remission criteria for lymphoma released by International Working Group (IWG) in 1999 and divided into four categories: complete remission (CR), complete remission uncertain $(\mathrm{CRu})$, partial response (PR) and progressive disease (PD).

\section{Follow-up and survival evaluation}

Patients were followed up through outpatient service, telephone or letter till June 30, 2012. The overall survival was the interval between the time of diagnosis and the time of death or last follow-up. Survival evaluation adopted 2-year overall survival (OS) rate after treatment and its correlation with clinicopathological features was studied. 


\section{Statistical analysis}

Data were processed using SPSS19.0 statistical software. Survival rate was estimated by Kaplan-Meier method and compared by Log-rank test. The difference was statistically significant when $\mathrm{P}<0.05$. Multivariate analysis was conducted by COX proportional hazard model and RR value was applied to describes the results (RR of $0.9-1$ or 1.0-1.1 indicated that the disease was not correlated with exposure factors; RR of $0.7-0.8$ or 1.2-1.4 indicated a weak correlation between exposure factors and the disease; RR of $0.4-0.6$ or 1.5-2.9 indicated a moderate correlation; RR $0.1-0.3$ or $3.0-9.0$ indicated a strong correlation; $\mathrm{RR}<0.1$ or $\mathrm{RR}>10$ indicated a very strong correlation).

\section{RESULTS}

\section{Basic clinical data}

Among the 18 cases of ENKCL, 13 patients survived and 5 died by June 2012, the end of the follow-up. All 5 deaths resulted from relapse or progression of the disease. The median survival time was 28.60 months, the average survival time was 28.18 months, and 2-year survival rate was $71.8 \%$ (Figure 1). 


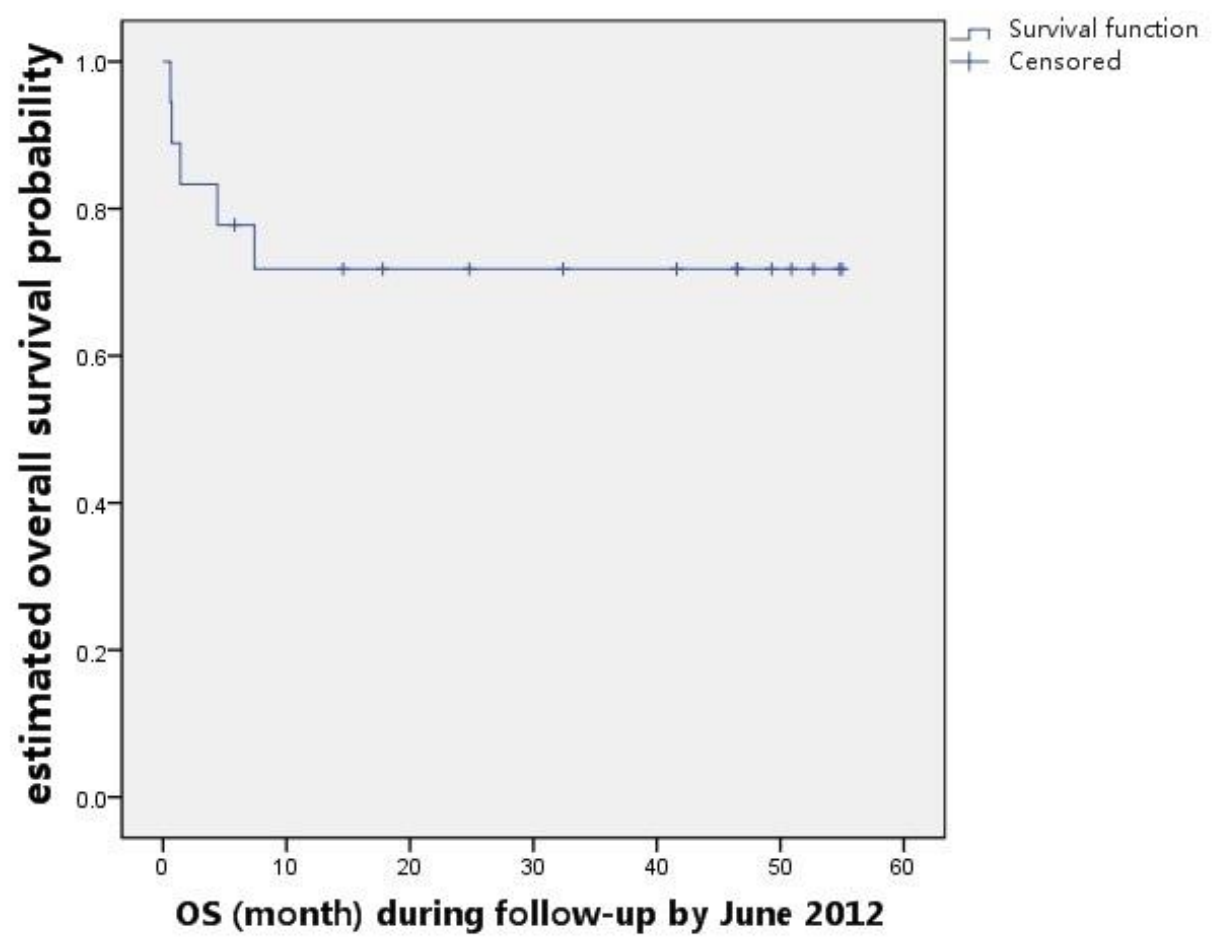

Figure 1. Survival curves of 18 ENKCL patients

The age of the 18 ENKCL patients ranged from 20 to 69 years, with an average age of 41.3, among which 16 patients were younger than 60 and 2 were older than 60 . Male/female ratio was 7/1. There were 9 Ann Arbor Stage I cases, 2 Stage II cases, 1 Stage III case and 6 Stage IV cases, 11 cases of ECOG PS score $<2$ and 7 cases of ECOG PS score $\geq 2,10$ cases without B symptoms and 8 cases with $\mathrm{B}$ symptoms, 13 cases without bone marrow infiltration and 5 cases with bone marrow infiltration, 11 cases of IPI $\leq 2$ and 7 cases of IPI $>2$, 11 cases of white blood cell count before treatment $\geq 4.0 \times 10^{9} / \mathrm{L} 11$ cases and 7 cases of white blood cell count $<4.0 \times 10^{9} / \mathrm{L}, 12$ cases of hemoglobin value $\geq 120 \mathrm{~g} / \mathrm{L}$ and 6 cases of hemoglobin value $<120 \mathrm{~g} / \mathrm{L}, 14$ cases of albumin concentration $\geq 35 \mathrm{~g} / \mathrm{L}$ and 4 cases of albumin concentration $<35 \mathrm{~g} / \mathrm{L}$, and 4 cases with normal lactate dehydrogenase level and 10 cases with elevated lactate dehydrogenase level. The analysis of the 2-year OS rate of the above indicators was shown in Table 1. 
Table 1. Correlation between basic clinical data and prognosis of 18 ENKCL patients

\begin{tabular}{|c|c|c|c|c|}
\hline Factor & $\begin{array}{l}\text { Number } \\
\text { of cases }\end{array}$ & $\begin{array}{l}\text { Number } \\
\text { of death }\end{array}$ & $\begin{array}{c}2 \text {-year OS rate } \\
(\%)\end{array}$ & Pvalue \\
\hline Gender & & & & 1.00 \\
\hline Male & 14 & 4 & 71.4 & \\
\hline Female & 4 & 1 & 75.0 & \\
\hline Age & & & & 0.49 \\
\hline$\leq 60$ & 16 & 4 & 74.5 & \\
\hline$>60$ & 2 & 1 & 50.0 & \\
\hline Ann Arbor Stage & & & & 0.02 \\
\hline Stage I / II & 11 & 0 & 100 & \\
\hline Stage III/IV & 7 & 5 & 28.6 & \\
\hline $\begin{array}{l}\text { Number of sites of extranodal } \\
\text { involvement }\end{array}$ & & & & 0.022 \\
\hline$<2$ 个 & $\begin{array}{l}11 \\
7\end{array}$ & $\begin{array}{l}2 \\
3\end{array}$ & $\begin{array}{l}91.7 \\
33.3\end{array}$ & \\
\hline$\geq 2$ 个 & & & & \\
\hline PS score & & & & 0.022 \\
\hline$<2$ & $\begin{array}{l}12 \\
6\end{array}$ & $\begin{array}{l}1 \\
4\end{array}$ & $\begin{array}{l}90.9 \\
33.3\end{array}$ & \\
\hline$\geq 2$ & & & & \\
\hline IPI score & & & & 0.002 \\
\hline$\leq 2$ & 11 & 0 & 100 & \\
\hline$>2$ & 7 & 5 & 28.6 & \\
\hline B symptoms & & & & 0.608 \\
\hline No & 10 & 2 & 100 & \\
\hline Yes & 8 & 3 & 50.0 & \\
\hline Bone barrow infiltration & & & & 0.008 \\
\hline No & 13 & 1 & 92.3 & \\
\hline Yes & 5 & 4 & 20.0 & \\
\hline WBC & & & & 0.047 \\
\hline$\geq 4.0 \times 10^{9} / \mathrm{L}$ & 11 & 1 & 90.9 & \\
\hline$<4.0 \times 10^{9} / \mathrm{L}$ & 7 & 4 & 42.9 & \\
\hline $\mathrm{LDH}$ & & & & 0.036 \\
\hline Normal & 8 & 0 & 100.0 & \\
\hline
\end{tabular}




\begin{tabular}{lllll}
\hline Elevated & 10 & 5 & 48.0 & \\
$\mathrm{HB}$ & & & & 0.022 \\
$\geq 120 \mathrm{~g} / \mathrm{L}$ & 12 & 1 & 90.9 & \\
$<120 \mathrm{~g} / \mathrm{L}$ & 6 & 4 & 33.3 & 0.533 \\
$\mathrm{ALB}$ & & & & \\
$\geq 30 \mathrm{~g} / \mathrm{L}$ & 14 & 3 & 77.9 & \\
$<30 \mathrm{~g} / \mathrm{L}$ & 4 & 2 & 50.0 & \\
\hline
\end{tabular}

\section{Correlation between efficacy of first treatment and prognosis}

18 ENKCL patients received chemotherapy alone, radiotherapy alone or combined treatment.

After first treatment, 11 patients achieved complete remission and 7 did not, with 2-year OS rates of $100 \%$ and $21.4 \%$ respectively. The difference presented statistical significance $(\mathrm{P}=$ $0.002<0.05)$ (Figure 2).

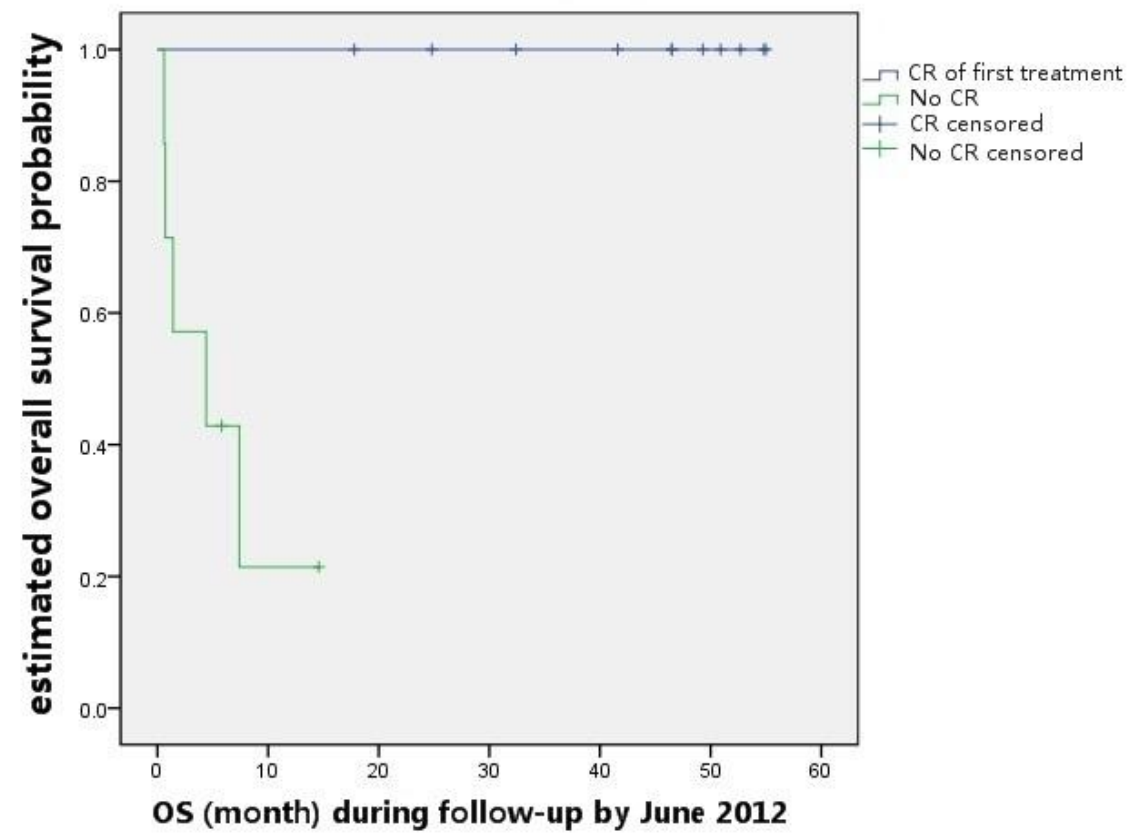

Figure 2. Survival curves of correlation between efficacy of first treatment and prognosis of $18 \mathrm{ENKCL}$ patients

\section{Correlation between first chemotherapy and prognosis}

15 out of 18 ENKCL patients received chemotherapy alone or combined treatment of 
radiotherapy or chemotherapy. 7 patients were treated with classic first-line CHOP regimen and 8 with the others. The 2-year OS rates were $85.7 \%$ and $46.9 \%$ respectively and showed no statistically significant difference $(\mathrm{P}=0.282>0.05)$ [Figure 3].

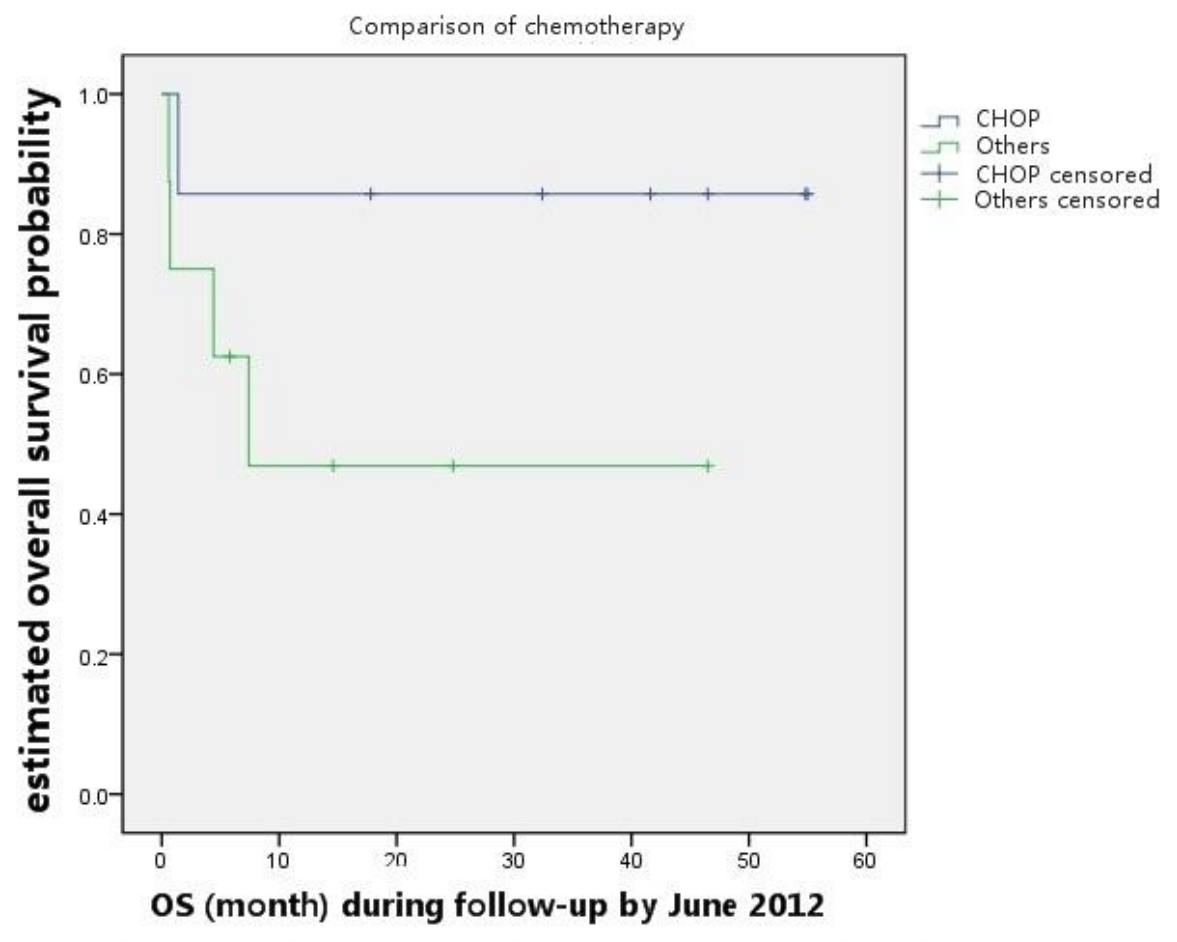

Figure 3. Survival curves of correlation between first-line chemotherapy and prognosis of 18 patients

\section{Correlation between cycles of chemotherapy and prognosis}

8 out of 18 ENKCL patients received chemotherapy alone, among which 4 cases applied 1-2 cycles of chemotherapy and 4 applied 2 cycles or above. The 2 -year OS rates were $25.0 \%$ and $75 \%$ respectively and indicated no statistically significant difference $(P=0.486>0.05)$ [Figure 4]. 


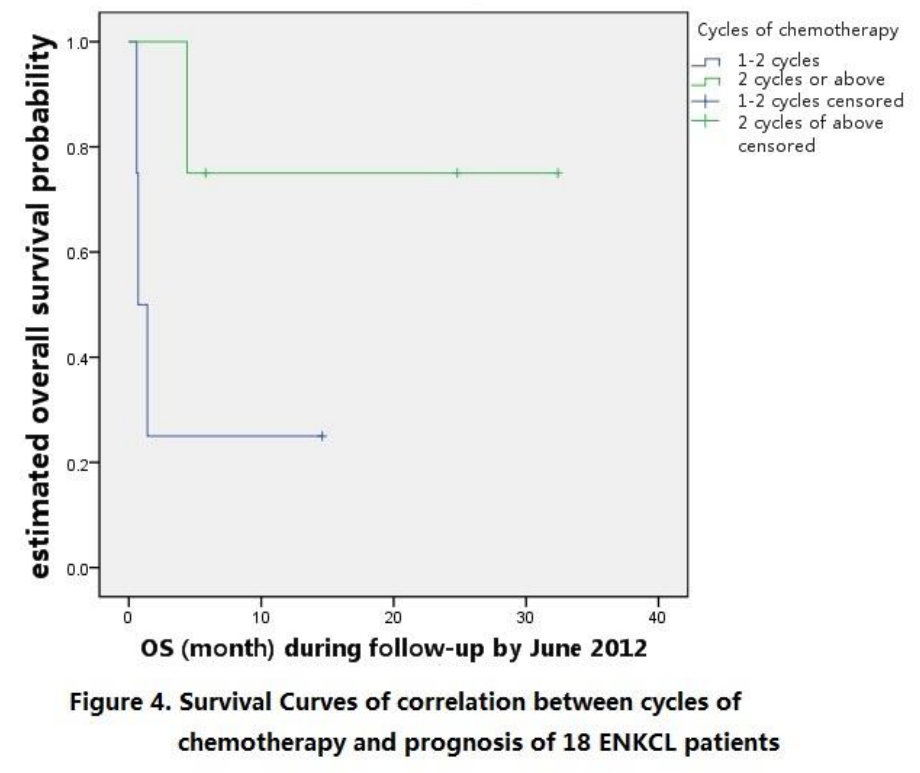

\section{Pathological diagnosis and immunohistochemical results}

Observation of pathological tissue complied with the heterogeneity of ENKCL. Mucous membranes often showed ulcers and diffuse abnormal lymphocytic infiltration, often damaging inherent mucosal gland. Tumor cells invaded the walls of small blood vessels or perivascular tissue, causing widespread coagulation necrosis (11).Immunohistochemical results suggested 16 cases of CD56-positive and 2 cases of CD56-negative. However, their $\mathrm{CD}_{3 \varepsilon}$ and TIA-1 expressions were positive. They complied with ENKCL features in accordance with morphology and WHO diagnostic criteria.

\section{Correlation between lymphocyte phenotype and prognosis}

Out of 18 ENKCL cases, 8 had NK cell phenotype and 10 had T cell phenotype, with 2-year OS rates of $100 \%$ and $50.0 \%$ respectively, which was statistically significant $(\mathrm{P}=0.036$ $<0.05)$ [Figure 5]. 


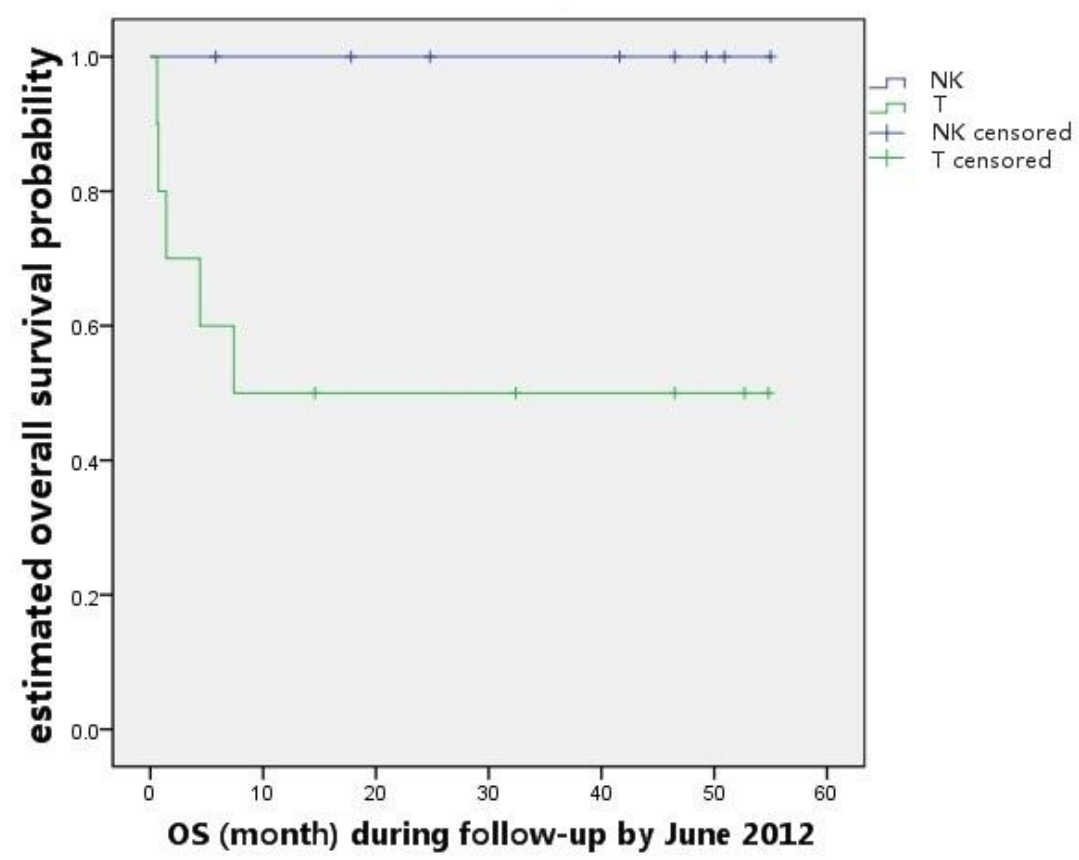

Figure 5. Survival Curve of correlation between lymphocyte phenotvpe and proqnosis of $18 \mathrm{ENKCL}$ patients

\section{Correlation between $\mathrm{CD}_{56}$ expression and prognosis}

Immunohistochemical tests found 11 cases of no negative expression and low expression (“+”) and 7 cases of moderate (“++") and high expression (“+++"), with 2-year OS rate of $90.9 \%$ and $42.9 \%$ respectively, suggesting statistically significant difference. Survival curves were shown in Figure 6. 


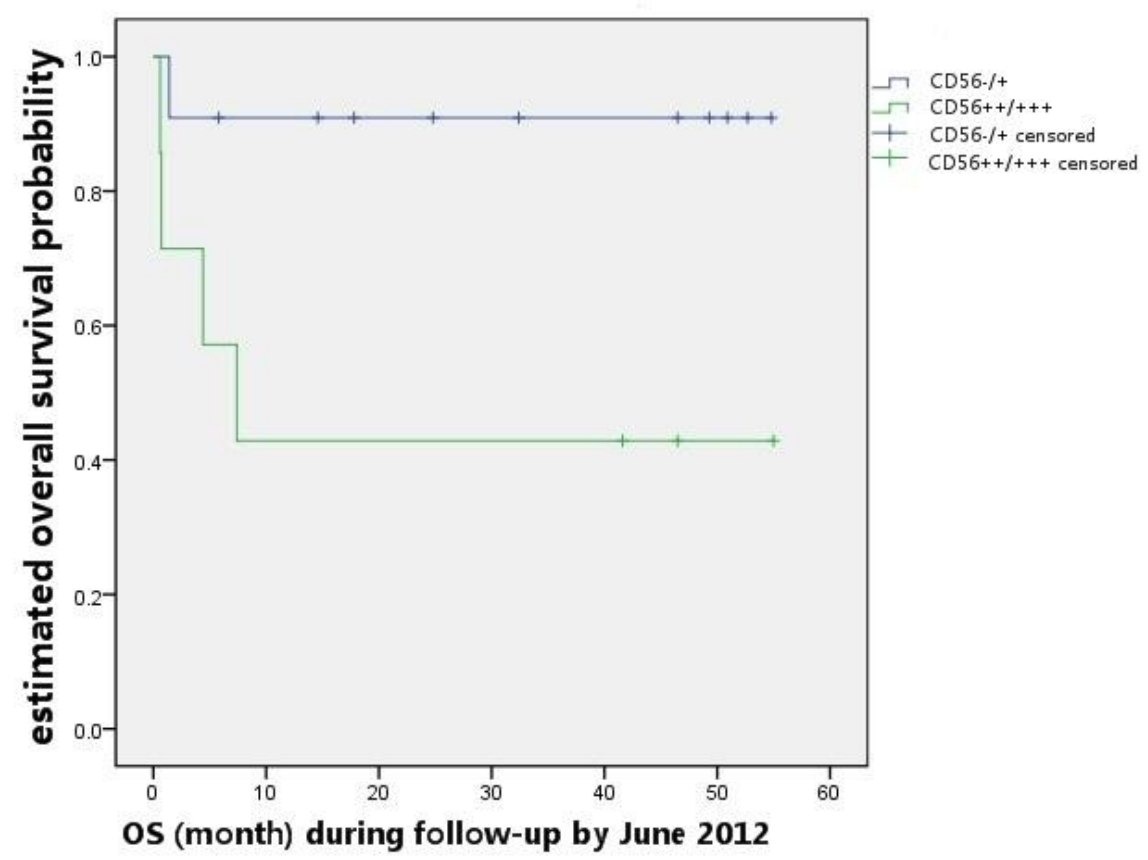

Figure 6. Survival curves of correlation between CD56 expression and prognosis of $18 \mathrm{ENKCL}$ patients

\section{between Ki-67 expression and prognosis}

Immunohistochemiscal results of 18 ENKCL cases showed 14 cases of Ki-67 proliferation index $\leq 30 \%$ and 4 cases of $\mathrm{Ki}-67 \geq 30 \%$. 2-year OS rates were $90.9 \%$ and $42.9 \%$ respectively and the difference was statistically significant $(\mathrm{P}=0.047<0.05)$ [Figure 7]. 


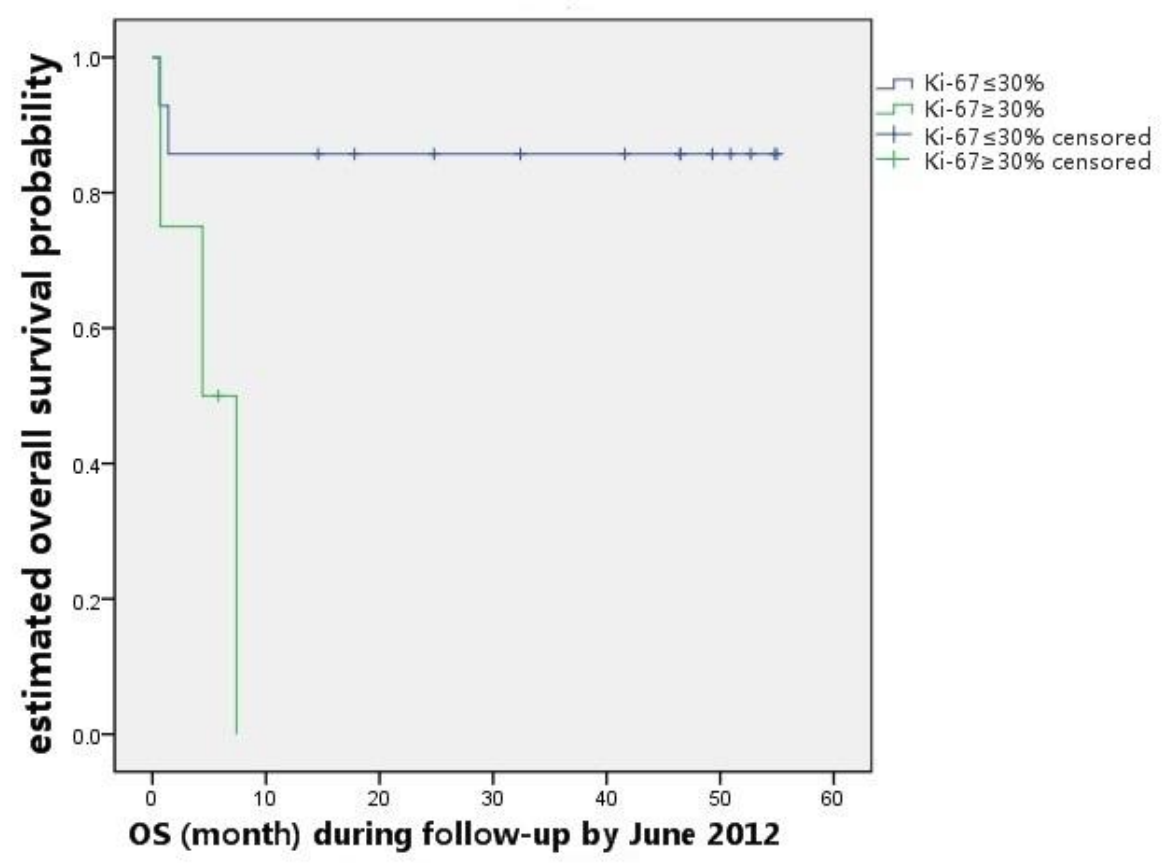

Figure 7. Survival curves of correlation between Ki-67 expression and prognosis of 18 patients

\section{Multivariate analysis of ENKCL prognosis}

We conducted COX regression analysis in the 18 cases, ECOG PS score, the number of extranodal involvement sites, IPI score, hemoglobin value before treatment, lactate dehydrogenase level, first treatment efficacy, CD56 and Ki-67 expression and lymphocyte phenotype showed a strong correlation with prognosis and survival (RR value: 284.641, 0.022806.740, 0.346, 0, 63525.14, 0.322, 0.273, 6.654), while Ann Arbor staging, bone marrow infiltration, white blood cell count before treatment showed $\mathrm{m}$ correlation with prognosis and survival (RR value: $2.887,2.875,1.848$ ).

\section{DISCUSSION}

Extranodal NK/T cell lymphoma, nasal type (ENKCL), mostly occurs in the nose and is 
characterized by aggressive and destructive nasal and mid-facial necrotizing lesions with frequent blood vessel involvement. It features a unique clinical presentation that includes fever, cachexia, rapid progression, and high mortality. Many related studies have been reported in recent years. However, the exact clinical features, prognostic factors, pathogenesis, and hence the optimal treatment paradigms of ENKCL remain unclear and consequently undeveloped. Therefore, studies regarding the clinical and pathological features and prognostic factors of ENKCL are meaningful.

Previous studies regarding the clinical pathological features and prognostic factors of ENKCL have mostly focused on the International Prognostic Index (IPI), which includes the age, Ann Arbor staging, Eastern Cooperative Oncology Group performance status (ECOG PS) score, number of extranodal involvement sites, and serum lactate dehydrogenase levels before treatment. However, long-term practice shows that the IPI score has certain limitations in clinical applications. In 1993, the IPI score was established in the retrospective study of cases dominated by B-cell lymphoma, which is quite different from ENKCL with regard to the origin and biological characteristics, indicating that the IPI score might be not suitable for ENKCL; therefore, some scholars continue to debate the significance of the IPI score for ENKCL prognosis $(12,13)$. In 2006, Lee et al. (14), in a multicenter study of prognostic factors of nasal NK/T cell lymphoma (262 cases), suggested that B symptoms, Ann Arbor staging, LDH levels, and regional lymph node involvement are independent prognostic factors with better prognostic outcomes than the IPI score. In 2011, the United States National Comprehensive Cancer Network (NCCN) guidelines revealed that in addition to the IPI score, tumor invasion, the Ki-67 tumor cell proliferation index, and an Epstein-Barr virus 
(EBV) DNA count greater than $6.1 \times 10^{9} / \mathrm{L}$ should also be included as risk factors for ENKCL. Recently, Huang et al. $(15,16)$ found that drug resistance, absolute lymphocyte counts, $\beta$-microglobulin expression, and disease progression are also important independent prognostic indicators. Therefore, some researchers believe that it is necessary to investigate IPI score-independent prognostic factors. Consistent with studies by Lee and Wang et al. (17), this study also confirmed that Ann Arbor staging, the ECOG PS score, sites of extranodal involvement, presence or absence of bone marrow infiltration, IPI scores, and LDH levels are independent prognostic factors for ENKCL. In addition, our study found that the blood leukocyte count before treatment and the hemoglobin $(\mathrm{Hb})$ level are independent prognostic factors for ENKCL. As early as 1998, Hasenclever et al. (18) reported that the Hb level before treatment was an independent prognostic factor in patients with advanced Hodgkin's lymphoma. In 2004, Solal-Celigny et al. (19) confirmed the relationship between Hb level and prognosis in patients with follicular non-Hodgkin's lymphoma. Many scholars believed that hypoxic cells were insensitive to radiotherapy and chemotherapy and that the poor effects of radiotherapy and chemotherapy in patients with low $\mathrm{Hb}$ levels might be related to the low oxygen concentration and increased number of hypoxic cells in the local tumor (20). The univariate analysis showed that age and sex had no significant effects on survival in this study. In contrast, others found a positive correlation between age and survival. The difference might result from the different age stratification of the selected subjects in different studies. B symptoms are often considered to be a poor prognostic factor in patients with lymphoma, probably because B symptoms (e.g., unexplained fever and night sweats) are related to an increase in tumor-associated factors (21). Similar to another study (22), our study also 
confirmed that B symptoms were not related to prognosis in ENKCL patients. Therefore, the effects of B symptoms on ENKCL prognosis require further study. The results herein regarding prealbumin concentrations were consistent with previous studies in which the albumin concentration after treatment was not found to be a poor prognostic factor of ENKCL (23). Furthermore, we found that after the initial course of chemotherapy, radiotherapy, or combination therapy, the 2-year overall survival rate in patients with complete disease remission was significantly increased in comparison to that of patients without complete disease remission, indicating that the efficacy of the initial treatment was important to the prognosis, which was consistent with our conventional understanding (24). Similar to the findings of other researchers that drugs such as gemcitabine and L-asparaginase can only reduce the side effects of chemotherapy rather than prolong survival, we found that the overall survival of patients who were treated with chemotherapy or a combination therapy of radiotherapy and chemotherapy was not affected by the use of different first-line regimens such as classical CHOP. Total survival did not significantly differ between patients who received more than 2 cycles of chemotherapy and those who received 1 or 2 cycles of chemotherapy but did improve as the chemotherapy cycle number increased. Survival was likely related to a short follow-up time or a small number of cases. In 2013, Lin Yutong et al., in a prospective phase II/III study, randomly selected subjects from among 109 patients with early-stage nasal NK/T cell lymphoma to receive either CID-ATT (CHOP-B, IMVD, and DHAP) or CHOP induction chemotherapy, followed by radiotherapy, with a median follow-up of 40.3 months, and found that the 5-year OS, PFS, and CR rates in the CID-ATT group were significantly better than those of the CHOP group, suggesting that it was 
potentially beneficial to apply existing chemotherapeutic drugs and innovative drug combinations to treat ENKCL. In recent years, autologous or allogeneic hematopoietic stem cell transplantation has been used in the clinic. Studies $(25,26)$ have demonstrated that allogeneic hematopoietic stem cell transplantation is among the curative choices available for adverse prognoses of ENKCL in young patients and thus provides a promising therapeutic strategy.

It is not known whether histopathological findings are as helpful as clinical results in the development of individual treatment regimes and assessments of clinical effects and prognosis. Tang et al.reported that large cell domination, vascular invasion, and focal necrosis are poor prognostic factors of ENKCL. In contrast, Wang et al. demonstrated that cell size, vascular invasion, and focal necrosis were not related to ENKCL prognosis. These factors, including EBV-encoded RNA (EBER) levels, were not analyzed in this study but should be verified in future studies. ENKCL might derive from precursor cells with the potential for NK and T-lymphocyte differentiation; hence, patients with ENKCL are often divided into either the NK- or T-cell phenotype based on immunohistochemical analysis. Following this classification, 18 patients in our study could be divided into 8 NK-cell phenotype cases and 10 T-cell phenotype cases. The 2-year OS rate for the NK-cell phenotype was $100 \%$, which was significantly different from the $50.0 \%$ rate for the T-cell phenotype. This finding indicated that the T-cell phenotype had a worse prognosis than did the NK-cell phenotype. CD56 is a glycoprotein that comprises polypeptide chains and is mainly expressed on the surfaces of NK cells. CD56 also contains polysialic acid units that can form a carbohydrate barrier that reduces contact with the extracellular medium and decreases cell adhesion, which 
might be associated with tumor invasion. Reportedly, CD56 can lead to the firm adhesion of tumor cells to blood vessel walls, thus damaging the blood vessels, and higher levels of CD56 expression can lead to more aggressive tumor invasion and shorter survival, compared with lower levels of CD56 expression (27). Anti-CD56 drugs can target tumors by specifically binding to CD56 and might be effective against ENKCL, suggesting that CD56 is important in ENKCL prognosis (28).

In this study, an immunohistochemical analysis revealed no negative CD56 expression or low expression ("+") in 11 cases and high CD56 expression (“++" or "+++") in 7 cases. Moreover, the 2-year OS rate of the former group was significantly higher than that of the latter, indicating that CD56 should be considered a prognostic indicator for ENKCL. $\mathrm{Ki}-67$ is a nuclear antigen present in proliferated cells, and its expression is closely related to cell mitosis. Ki-67 expression occurs during the late G1 phase of the cell cycle, increases during the $\mathrm{S}$ and $\mathrm{G} 2$ phases, peaks during the $\mathrm{M}$ phase, and quickly disappears or loses antigenicity post mitosis. Ki-67 is not expressed during DNA repair, has a half-life of only 1 $\mathrm{h}$ or shorter, and is considered among the most reliable indicators of tumor cell proliferation of tumor cells but has been classified as a poor prognostic factor by the NCCN (29). In fact, tumors are usually sensitive to chemotherapy; however, there is a high proportion of proliferated cells with high Ki-67 expression in tumors because of the short doubling time, which can result in a high tumor burden; hence, Ki-67-based prognoses are poor (30). The univariate analysis herein showed that the survival of patients with low Ki-67 expression levels was significantly longer than that of patients with high Ki-67 expression levels, and the latter group presented with the characteristics of a low remission rate, recurrence, and poor 
prognosis during ENKCL treatment.

In this study, we included information regarding the rapid development of ENKCL lesions, poor prognosis, Ann Arbor staging, ECOG PS scores, the numbers of sites of extranodal involvement, bone marrow infiltration, IPI scores, white blood cell counts before treatment, Hb levels, LDH levels, first-line treatment effects, CD56 and Ki-67 expression, and lymphocyte phenotypes in 18 patients with ENKCL in a COX proportional hazard model for a multivariate analysis. The results showed that the survival and prognosis of ENKCL patients correlated strongly with the ECOG PS score, number of extranodal involvement sites, IPI score, $\mathrm{Hb}$ levels before treatment, LDH levels, first-line treatment effect, CD56 and Ki-67 expression, and lymphocyte phenotype. The analysis indicated that these factors could greatly affect the survival and prognosis of ENKCL patients. Meanwhile, the tumor stage, bone marrow infiltration, and white blood cell counts before treatment also correlated with the survival and prognosis of ENKCL patients, suggesting that these indicators were also important to the survival and prognosis of ENKCL patients.

In summary, the current study regarding the clinicopathological characteristics and prognostic factors of ENKCL could provide valuable information for the development of individualized treatment strategies, especially for refractory and relapsed ENKCL. Hence, the results herein should be verified in future studies with a large number of cases and a long-term follow-up. Given the better understanding of ENKCL clinical pathology-related cell genetics and pathogenesis and the application of gene chip technology, it is hoped that more clinicopathologically significant prognostic indicators for ENKCL can be identified (31, 32). 


\section{REFERENCES}

1. Siegel R, Naishadham D, Jemal A. Cancer statistics 2012. CA Cancer J Clin. 2012; 62:10-29.

2. Kwong YL.The diagnosis and management of extranodal NK/T-cell lymphoma, nasal-type and aggressive NK-cell leukemia.J Clin Exp Hematop. 2011;51:21-28.

3. Pongpruttipan T, Sukpanichnant S, Assanasen T, et al. Extranodal NK/T-cell lymphoma, nasal type, includes cases of natural killer cell and $\alpha \beta, \gamma \delta$, and $\alpha \beta / \gamma \delta$ T-cell origin: a comprehensive clinicopathologic and phenotypic study. Am J Surg Pathol. 2012;36:481-499.

4. Park JH, Shin HT, Lee DY, et al. Extranodal natural killer (NK)/T-cell lymphoma presenting with recurrent lip swelling.Int J Dermatol. 2013;52:763-765.

5. Chan JKC, Jaffe ES, RaIfkiaer E. ExtranodaI NK/T ceII Iymphoma, nasaI type(A).In:Jaffe ES, Vardiman JW. Pathology and genetics of tumors of haematopoietic and Iymphoid tissues (M). Lyon: Inter-nationaI Agency for Research on Cancer ( IARC ) Press,2001; pp:204-407.

6. Yasuda H, Sugimoto K, Imai H, et al. Expression levels of apoptosis-related proteinsand Ki-67 in nasal NK/T-cell lymphoma. Eur J Haematol, 2009;82:39-45.

7. Obermann EC, Eppenberger-Castori S, Tapia C. Assessment of proliferation: core biopsy or resection specimen? Discrepancies in breast cancer with low and high proliferation. Pathologe. 2012;33:245-250.

8. Lorenzen J, Liu WP, Gi GD, et al.Nasal T/NK cell lymphoma: a clinico pathologic study of 30 west Chinese patients with special reference to proliferation and apoptosis.Leuk Lymphoma. 1996;23:593-602.

9. Takahashi N, Miura I, Chubachi A, et al. A clinicopathological study of 20 patients with T/natural killer (NK)-cell lymphoma-associated hemophagocytic syndrome with special reference to nasal and nasal-type NK/T-cell lymphoma.Int $\mathbf{J}$ Hematol. 2001;74:303-308.

10. Woo JS , Kim JM, Lee SH, et al. Clinical analysis of extranodal non Hodgkin's lymphoma in the sinonasal tract. Eur Arch Otorhinolaryngo.2004;261:197-201. 
11. Chim CS, Ma SY, Au WY, et al.Primary nasal natural killer cell lymphoma: long-term treatment outcome and relationship with the International Prognostic Index.Blood. 2004;103:216-221.

12. Kim WS, Song SY, Ahn YC, et al.CHOP followed by involved field radiation: is it optimal for localized nasal natural killer/T-cell lymphoma?Ann Oncol. 2001;12:349-352.

13. Lee J ,Suh C ,Park YH ,et al .Extranodal natural killer T-cell lymphoma ,nasal type : a prognostic model from a retrospective multicenter study. J Clin Oncol. 2006;24:612-618 .

14. Huang JJ, Jiang WQ, Lin TY, et al. Absolute lymphocyte count is a novel prognostic indicator in extranodal natural killer/T-cell lymphoma, nasal type.Ann Oncol. 2011;22:149-155.

15. Li ZM, Zhu YJ, Sun J, Xia Y, Huang JJ, Zou BY, Lin TY, Huang HQ, Jiang WQ.Serum beta2-microglobin is a predictor of prognosis in patients with upper aerodigestive tract NK/T-cell lymphoma.Ann Hematol. 2012;91:1265-1270.

16. Wang BY, Hong XN, Yin JL, et al. Prognostic predictors of nasal NK/T cell lymphoma detected by immunohistochemical staining. Zhonghua Zhong Liu Za Zhi. 2006;28:523-525.

17. Hasenclever D, Diehl V. A prognostic score for advanced Hodgkin's disease. International Prognostic Factors Project on Advanced Hodgkin's Disease. N Engl J Med. 1998;339:1506-1514.

18. Solal-Celigny P, Roy P, Colombat P, et al. Follicular lymphoma international prognostic index . Blood. 2004,104:1258-1265 .

19. Ribrag V, E11 Hajj M, JaIlot F, et a1. Eally locoregional high-dose radiotllempy is associated with long-ternl disease control in localized primary angiocentric lymphoma of the nose and nasopharynx. Leukemia. 2001;15:1123-1126.

20. Lee BN, Dantzer R, Langley $\mathrm{KE}$, et a1.A cytokine-based neuroimmunologic mechanism of cancer-related symptoms. Neuroimmunomodulation. 2004; 11: 
279-292.

21. Zhang YJ, Hu WH, Liu H, et al. Clinical features and prognosis of nasal type NK/T cell lymphoma. Zhonghua Zhong Liu Za Zhi. 2006;28:50-53.

22. Xu Gang, Wang Huaifu, Du Zexiu, et al. Prognostic factors of nasal NK/T-cell lymphoma. CHIN ARCH OTOLARYNGOL HEAD NECK SURG. 2007,14:393-396

23. Han DM, Chen XQ, Bai QX. Exploration of prognostic factors in extranodal nasal-type NK/T cell lymphoma. Zhonghua Zhong Liu Za Zhi. 2006;28:137.

24. Yokoyama H, Yamada MF , Ishizawa K , et al . Successful treatment of advanced extranodal NK/T cell lymphoma with unrelated cord blood transplantation. Tohoku J Exp Med. 2007;211:395-399 .

25. Li M, Gao C, Li H, Wan g Z, et al. Allogeneic haematopoietic stem cell transplantation as a salvage strategy for relapsed or refractory nasal NK/T-cell lymphoma. Med Oncol. 2011;28: 840-845.

26. Li CC, Tien HF, Tang JL,et al.Treatment outcome and pattern of failure in 77 patients with sinonasal natural killer/T-cell or T-cell lymphoma. Cancer. 2004;100:366-375.

27. Ishitsuka K, Jimi S, Goldmacher VS, et al.Targeting CD56 by the maytansinoid immunoconjugate IMGN901 (huN901-DM1): a potential therapeutic modality implication against natural killer/T cell malignancy. Br J Haematol. 2008; 141: $129-31$.

28. Freeman A, Hamid S, Morris L, et al.Improved detection of hepatocyte proliferation using antibody to the pre-replication complex: an association with hepatic fibrosis and viral replication in chronic hepatitis $\mathrm{C}$ virus infection. J Viral Hepat. $2003 ; 10: 345-350$.

29. Kim SJ, Kim BS, Choi CW,et al. Ki-67 expression is predictive of prognosis in patients with stage I/II extranodal NK/T-cell lymphoma, nasal type.Ann Oncol. 2007; 18: 1382-87.

30. Jaffe ES.Lymphoid lesions of the head and neck: a model of lymphocyte homing and lymphomagenesis. Mod Pathol. 2002; 15: 255-63. 
31. Wang L, Wang ZH, Chen XQ, et al.First-line combination of gemcitabine, oxaliplatin, and L-asparaginase (GELOX) followed by involved-field radiation therapy for patients with stage IE/IIE extranodal natural killer/T-cell lymphoma. Cancer. 2013; 119: 348-55.

32. Kim SJ, Yoon DH, Kang HJ, et al.Bortezomib in combination with CHOP as first-line treatment for patients with stage III/IV peripheral T-cell lymphomas: a multicentre, single-arm, phase 2 trial. Eur J Cancer. 2012; 48: 3223-31. 